\title{
Unity Is Strength: Coupling Media for Thematic Segmentation
}

\author{
Dalila Mekhaldi, Denis Lalanne, and Rolf Ingold \\ Université de Fribourg, Chemin de musée 3, CH-1700 Fribourg \\ \{dalila.mekhaldi, denis.lalanne,rolf.ingold\}@unifr.ch
}

\begin{abstract}
In this paper we present the preliminary results and the evaluation of a combined thematic segmentation of (a) meeting documents and (b) meeting speech transcript. Our approach is based on a clustering method applied on a $2 \mathrm{D}$ representation of the thematic alignment, and then the projection of the extracted clusters on each axis, corresponding to meeting documents and the speech transcript. Finally, our bi-modal thematic segmentation method is evaluated, in regards to a mono-modal segmentation method (TextTiling).
\end{abstract}

\section{Introduction}

In the context of multimodal applications, especially meeting recordings and lectures, research are in hand, in order to establish temporal links between the various modalities, mainly between documents and meetings dialogs [5]. Our viewpoint is that bridging temporal links between these two modalities may be attained once their thematic links, i.e. their thematic alignment, are established.

The document/speech thematic alignment and the thematic segmentation are closely related. The thematic alignment is building thematic links between documents and speech units, which are semantically close. While thematic segmentation builds thematic links between units of a unique modality (document or speech). Thematic segmentation is thus an intra-modal segmentation, while thematic alignment is an inter-modal segmentation. Since the preliminary evaluation we have performed on state-of-the-art, thematic segmentation methods did not show good results, our assumption is that an inter-modal segmentation will be more efficient and will benefit from the various modalities information. In this article, we present briefly our bi-modal thematic segmentation method and its projection to each modality. A preliminary evaluation shows that our bi-modal segmentation is more efficient than a mono-modal segmentation.

\section{Thematic Alignment vs. Thematic segmentation}

Our document/speech alignment takes as input the speech transcript of a meeting and the documents related to the meeting, and generates a set of aligned pairs (document units, speech units) [5]. Currently, we are focusing on press reviews, where many speakers discuss a daily newspaper cover page. 


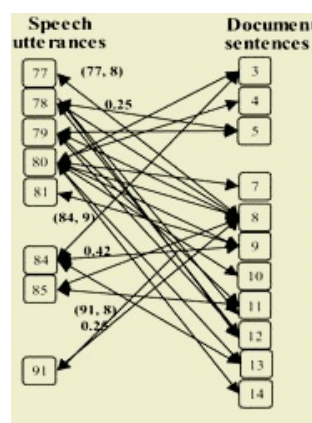

a.

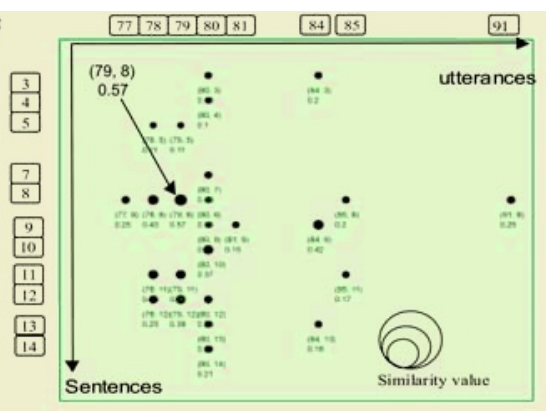

b.

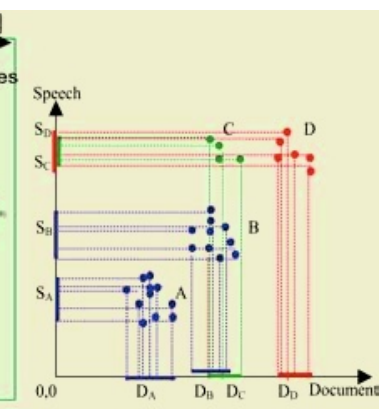

c.

Fig. 1. a. Bi-graph representing the thematic alignment b. $2 \mathrm{D}$ representation of the bi-graph c. Clusters projection.

The information contained in the documents in PDF form, is first extracted and then automatically converted into a multi-layered structure (layout, logical and syntactical structures) [2]. The document logical structure is a hierarchical decomposition of the document into a set of labels. The logical structure of our newspaper cover page is a set of articles, where each article contains a title, content and an author, etc. However, the syntactical structure is a segmentation of the document into a set of textual components, e.g. sentences and paragraphs. From another side, the speech is currently manually transcribed, and is composed of thematic episodes, which contain many speakers' turns. Each turn contains at least one utterance, which is a homogeneous speaker part.

Once the document and the speech transcript structures are acquired, a matching process based on various similarity methods (Cosine, dice, Jaccard) is achieved between the various pairs (e.g. sentences with utterances, turns with logical blocks, etc.) [4]. Thus, for a given unit from the source file (document or speech), all the similar units in the target file are selected (figure 1.a).

This thematic alignment, which is a symmetrical relationship between the document and speech units, can be represented by a 2-dimensional graph, where each dimension represents a distinct modality (figure 1.b). Each node in this representation is a relationship between the document and speech units (e.g. utterance 79 with sentence 8 has a similarity value of 0.57 ), and the node size represents their similarity value. Starting from this $2 \mathrm{D}$ representation, a clustering process based on an improved $K$-means method has been applied in order to bring to light the denser regions, that we believe that may represent the various meeting topics. This clustering method was enriched by a filtering step of the weak densities clusters, by considering the clusters size, the nodes weights and distances (e.g. Euclidean distance) from the clusters centroids.

Once the denser regions are computed, they are projected on each axis in order to highlight the mono-modal thematic segments. In figure 1.c the cluster $A$ corresponds to a document segment $D_{A}$ and a speech segment $S_{A}$. 
Table 1. Documents/Speech thematic segmentation evaluation.

\begin{tabular}{|c|c|c|c|c|c|c|c|c|c|c|c|c|c|c|c|}
\hline \multirow[b]{2}{*}{ Metrics } & \multicolumn{7}{|c|}{ Document } & \multicolumn{8}{|c|}{ Speech } \\
\hline & \begin{tabular}{l|l|}
$D_{1}$ & $D_{2}$ \\
\end{tabular} & $D_{3}$ & $D_{4}$ & $D_{5}$ & $\overline{D_{6}}$ & \begin{tabular}{|l|}
$D_{7}$ \\
\end{tabular} & 0 & N1 & $S_{2}$ & N3 & $S_{4}$ & $S_{5}$ & $\mathrm{~N} 6$ & $S_{7}$ & N8 \\
\hline ht & \begin{tabular}{|l|l|}
14 & .14 \\
\end{tabular} & 14 & .14 & 38 & .17 & .23 & 36 & .25 & .34 & .33 & .31 & .20 & .16 & .13 & \\
\hline & \begin{tabular}{l|l|}
82 & .74 \\
\end{tabular} & 82 & .82 & .60 & .78 & .67 & 64 & 18 & .69 & .68 & .67 & .79 & .81 & .85 & 8 \\
\hline$\overline{P_{k}}$ & \begin{tabular}{|l|l|}
.41 & .31 \\
\end{tabular} & .38 & \begin{tabular}{|l|}
.32 \\
\end{tabular} & .54 & .25 & \begin{tabular}{|l|}
.43 \\
\end{tabular} & .40 & .36 & .35 & .39 & .46 & .42 & .33 & .43 & .42 \\
\hline
\end{tabular}

Table 2. $P_{k}$ evaluation of a bi-modal method, comparing to a mono-modal method.

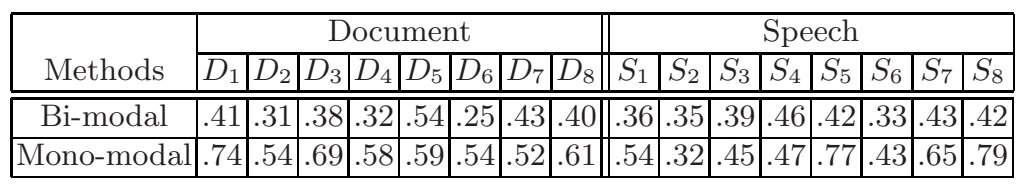

\subsection{Experimental Results}

Many metrics have been used in the evaluation of our method, in respect to a prepared manual ground truth: the entropy/purity and the $P_{k}$ (Beeferman) metric [1]. The entropy measures the disorder of segments. On the other hand, the purity measures the fraction of generated segments that does not contain incorrectly placed objects. For a perfect segmentation, the respective values for the two metrics are 0 and 1 . The $P_{k}$ metric measures the probability that a randomly chosen pairs of units at a distance of $\mathrm{k}$ units apart are inconsistently classified, with a value of 0 for a perfect segmentation. This metric is more adequate than a simple recall/precision that measures just the boundaries detection. For this experiment, the $\mathrm{k}$ parameter has been fixed to 4 units, which corresponds to the minimum size of a relevant thematic segment.

Table 1 shows the evaluation of the thematic segmentation of 8 meetings documents and speech transcripts. The generated entropy/purity values depend on the type of the meeting. Thus we distinguish two types:

1. If the speakers do not follow the linearized documents reading order, then the temporal indexes of the document segments are not adjacent. This reduces the number of overlapped segments, and as a result, it gives the satisfactory values for the entropy/purity (e.g. documents $D_{1}, D_{2}, D_{3}$ and $D_{4}$ ).

2. If the meeting is non-stereotyped, i.e. with numerous debates, then there are less overlapped segments (e.g. $S_{6}, S_{7}$ and $S_{8}$ ). This is due to the fact that the speech segments are well separated each one from the other. As results, their entropy/purity values are better, comparing to stereotyped meetings.

The $P_{k}$ evaluation is generally satisfactory (see Table 2), especially in comparison to the TextTiling[3] method. Our bi-modal segmentation method is more accurate in detecting the exact number of thematic segments, which is not the case for the TextTiling method that generates many extra segments. 


\subsection{Remarks}

During the segments extraction process, overlapping problems often occurred. This kind of problems happens when a unit is assigned to many segments, and it mainly appears in stereotyped meetings. The relationship between the overlapped segments can be one of two types: either one of them contains the other (e.g. in the figure 1.c, $S_{D}$ contains $S_{C}$ ), or they are partially overlapped (e.g. $D_{B}$ with $D_{C}$ ). Our contribution in resolving this problem is under work, and is based on the Gaussian probabilistic function. First, an overlapping coefficient is computed. Depending on this coefficient, the corresponding segments are merged, or considered as two distinctive segments, using the Gaussian probabilistic.

Other works are planned in order to improve this bi-modal thematic segmentation, such as the integration of the nodes weights in the clustering method, while computing the clusters centroids then while assigning the nodes to the clusters.

\section{Conclusion and Future Work}

This paper shows the results of the evaluation of a bi-modal thematic segmentation method, based on a preliminary thematic alignment of meetings documents with speech transcripts. The comparison of this method with a mono-modal method, i.e. TextTiling method, shows promising results, despite the overlapping problem that affects the segmentation, and should be resolved. The segmentation quality can be improved by considering the nodes weights earlier in the clustering process. Other prospects are foreseen, such as the combination with other alignments, for instance the speech turns with the documents logical units, or references to documents in meeting dialogs, citations, etc. In a long term, we plan to integrate all the various types of alignments in a single framework.

This preliminary evaluation makes us believe that coupling modalities, in this meeting documents and speech transcripts, should considerably improve each involved modality segmentation.

\section{References}

1. Beeferman D., Berger A., Lafferty J. (1999), Statistical Models for Text Segmentation, Machine Learning, Vol. 34(1/3), pp. 177-210.

2. Hadjar K., Rigamonti M., Lalanne D. and Ingold R., Xed: a new tool for eXtracting hidden structures from Electronic Documents, DIAL 2004, Palo Alto, California.

3. Hearst M., Multi-Paragraph Segmentation of Expository Text, In Proceedings of the ACL'94, Las Cruces, New Mexico.

4. Lalanne D., Mekhaldi D. and Ingold R. Talking about documents: revealing a missing link to multimedia meeting archives. Document Recognition and Retrieval XI, IS-T/SPIE's International Symposium on Electronic Imaging 2004, USA

5. Mekhaldi D., Lalanne D. and Ingold R., Thematic Alignment Of Recorded Speech With Documents,Proceedings of the 2003 ACM symposium on Document engineering, France 